\title{
NEP-WIDE POINT SOURCE CATALOG
}

\author{
Seong Jin Kim, Hyung Mok Lee, and NeP-Wide Team \\ Department of Physics and Astrnomy, Seoul National University, Seoul 151-747, Korea \\ E-mail: seongini@astro.snu.ac.kr \\ (Received July 01, 2012; Accepted August 03, 2012)
}

\begin{abstract}
We present a photometric catalog of infrared (IR) sources based on the North Ecliptic Pole Wide field (NEP-Wide) survey of AKARI, which covered a $5.4 \mathrm{deg}^{2}$ circular area centered on NEP. The catalog contains about 115,000 sources detected at the 9 IRC filter bands, comprehensively covering a wavelength range from 2 to $24 \mu \mathrm{m}$. This is a band-merged catalog including all of the photometry results from the supplementary optical data as well as the IRC bands. To validate a source at a given IRC band, we searched for counterparts in the other bands. The band-merging was done based on this cross-matching of the sources among the filter bands. The NIR sources without any counterpart in any other bands are finally excluded to avoid false objects.
\end{abstract}

Key words: methods: data analysis; infrared: galaxies; surveys; catalogs

\section{INTRODUCTION}

The NEP-Wide survey (Matsuhara et al., 2006) of AKARI covered about $5.4 \mathrm{deg}^{2}$ with a circular shape. Using nine photometric bands, comprehensively covering a wavelength range from 2 to $24 \mu \mathrm{m}$ (Murakami et al., 2007), the survey detected various types of galactic and extra-galactic sources (Lee et al., 2009; Kim et al., 2012). Together with the NEP-Deep survey (Wada et al., 2008; Takagi et al., 2012), the significance in astronomy of the public role of a reliable source catalog after such a survey mission can hardly be overemphasized. Therefore, we made extensive efforts in order to minimize false sources caused by various reasons.

Here we briefly describe the methodology used to validate the detected sources, and present an overview of the NEP-Wide point source catalog. More detailed description on the data reduction and the correction procedures can be found in Kim et al. (2012).

\section{CONFIRMATION OF DETECTED SOURCES}

To construct a reliable source catalog, we focused on the rejection of false objects by matching of the sources detected in one IRC band against those in all the other bands as shown in Fig. 1.

Ancillary optical data obtained at the CFHT (Hwang et al., 2007) and the Maidanak (Jeon et al., 2010) observatories and near-IR data at the KPNO are used for this procedure. The optical data are crucial for identifying the nature of the corresponding AKARI sources, since the stellarity parameters help to distinguish between stars and galaxies. $J, H$ data are used to bridge the AKARI/NIR data and the optical data.

The matching among the different wavebands is used for confirmation of the sources. As shown in Fig. 1, about 1,590 of the $N 2$ sources $(\sim 1.8 \%)$ do not have any counterpart in any of the other bands. In the $N 3$ band, about $3.9 \%$ of the sources remained unmatched to any other band data. All of these unmatched sources can not be confirmed and are likely to be false objects. We excluded these sources in the catalog. The fractions of sources without any counterparts in the MIR-S are $1.0 \%$ and $2.2 \%$ for the $S 9 W$ and $S 11$ respectively. For the MIR-L bands, about $7 \%$ and $10 \%$ of the sources in the $L 15$ and $L 18 W$ bands remained unmatched against those in other bands. 


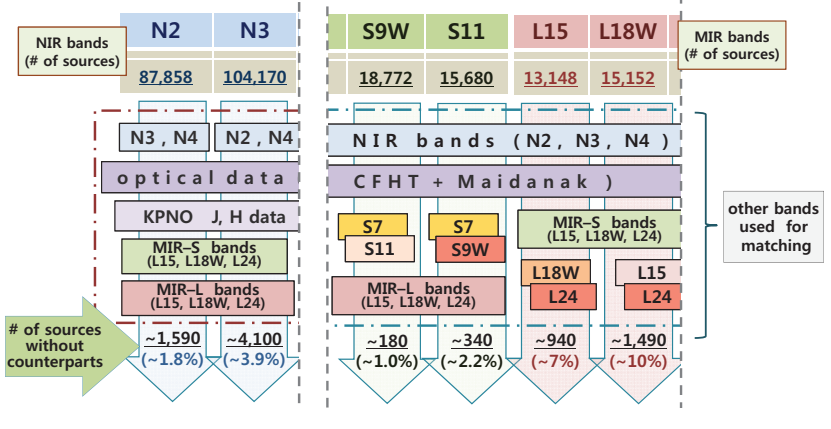

Fig. 1. Schematic overview of the matching procedures for the sources in AKARI bands. The boxes at the top of the diagram show the number of detected sources in each band. Under those boxes, the downward arrows denote the matching order.

\section{POINT SOURCE CATALOGUE}

In the AKARI/NEP-Wide data, the number of sources in the NIR bands are much larger than that of the MIR bands. Consequently, the majority of MIR sources have NIR counterparts, but not vice versa. Therefore, we proceed to the compilation of the matching results on the basis of the NIR framework.

After the source matching we generated separate catalogs for the nine IRC bands. At this point, each catalog contained the photometric information from other bands. We merged all these catalogs step by step. We made the NIR band catalog from the $N 2, N 3$, and $N 4$ single band catalogs by cross-matching the sources between the three bands, and where possible, with the optical data. MIR-S and MIR-L catalogs were also generated with the same method as that used for the NIR catalog. As shown in Fig. 2, the final band-merging was based on the NIR catalog, and the sources in the MIR-S and MIR-L catalogs were compiled by matching of the entries using corresponding source IDs. For registered sources in the catalog, if there is no detection in a certain band, we assigned the dummy value, 99.000. NIR sources having no counterpart in any other bands are excluded to avoid false objects caused by various artifacts. As explained, however, we carried out careful eye inspection on individual images of MIR sources having no counterparts before the exclusion.

The number of sources in this final catalog is about 114,800 . The contents included in the catalog are the identification (ID) numbers, the position (R. A and Dec.), magnitudes, RMS errors from the IRC as well as the ancillary data, and optical stellarity, and so on

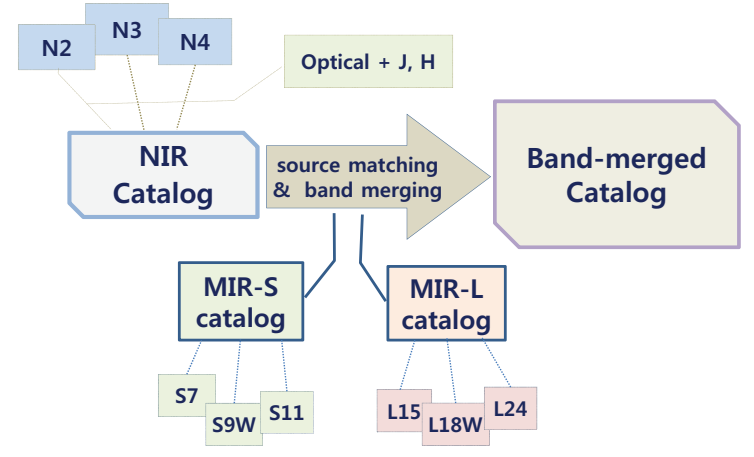

Fig. 2. Schematic diagram describing the band merging procedure. After the combination of the catalogs for each IRC channel, we compiled them into a band-merged catalog covering from optical $u^{*}$ to $L 24$ band. During this procedure, we checked the source IDs carefully in order to avoid duplication of the same entries.

(see Kim et al., 2012 for details).

\section{ACKNOWLEDGEMENTS}

This work is based on observations with AKARI, a JAXA project with the participation of ESA, universities and companies in Japan, Korea, the UK, and Netherlands. This work was supported by the Korean Research Foundation grant 2006-341-C00018.

\section{REFERENCES}

Hwang, N., et al., 2007, An Optical Source Catalogue of the North Ecliptic Pole Region, ApJS, 172, 583

Jeon, Y., et al., 2010, Optical Images and Source Catalogue of AKARI North Ecliptic Pole Wide Survey Field, ApJS, 190, 166

Kim, S. J., Lee, H. M., et al., 2012, North Ecliptic Pole Wide Field Survey of AKARI: Near- and Mid Infrared Source Catalog, A\&A, accepted

Lee, H. M., Kim, S. J., et al., 2009, North Ecliptic Pole Wide Field Survey of AKARI: Survey Strategy and Data Characteristics, PASJ, 61, 375

Matsuhara, H., et al., 2006, Deep Extragalactic Surveys around the Ecliptic Poles with AKARI, PASJ, 58, 673

Murakami, H., et al., 2007, The Infrared Astronomical Mission AKARI, PASJ, 59, S369

Takagi, T., et al. 2012, The AKARI NEP-Deep Survey: a Mid-Infrared Source Catalogue, A\&A, 537, A24

Wada, T., et al., 2008, AKARI/IRC Deep Survey in the NEP region, PASJ, 60, S517 